\title{
Desviadxs $^{1}$ : personas trans y sus discursos respecto a ocupaciones ${ }^{2}$
}

\author{
Deviatxs: trans people and their speeches in regard to occupations \\ Desviadx: pessoas trans e seus discursos sobre ocupações
}

\author{
Silvana Aravena Arroyo ${ }^{3}$ \\ Juan Pablo Contreras Briones ${ }^{4}$ \\ Mónica Palacios Tolvett ${ }^{5}$
}

Recibido: 15 de septiembre 2018 • Enviado para modificación: 18 de junio 2019 • Aceptado: 6 de agosto 2019

Aravena-Arroyo, S., Contreras-Briones, J.P. \& Palacios-Tolvett, M. (2019). Desviadxs: personas trans y sus discursos respecto a ocupaciones. Revista Ocupación Humana, 19 (1), 6- 21. doi: https://doi.org/10.25214/25907816.270

\section{RESUMEN}

Esta investigación propone reconocer discursos subalternos de personas transexuales-transgénero (trans) acerca de sus ocupaciones, visibilizando la diversidad de sus prácticas sociales. La metodología fue cualitativa, de carácter descriptivo-interpretativo. Se realizaron entrevistas en profundidad, semi-estructuradas, a cuatro personas mayores de 18 años, seleccionadas de manera intencionada. Se efectuó análisis de discurso, estableciendo tres ejes: autoreconocimiento; discurso y ocupación; práctica social y sus lugares de enunciación. Los resultados dan cuenta de que lxs sujetxs trans desarrollan su identidad desde estereotipos de género referentes a la dicotomía hombre-mujer, donde socialmente lo masculino presenta privilegios sobre lo femenino; así, se advierte que la heteronormatividad patriarcal está implícita en sus ocupaciones y en su vida cotidiana. Emergen discursos dominantes y subalternos, comprendidos desde lo bueno-aceptable y lo malo-fuera de la norma.

\section{PALABRAS CLAVE}

personas tránsgenero, heteronormatividad, ocupaciones, discurso

\footnotetext{
1 A lo largo del texto, Ixs autores deciden usar la x como lenguaje inclusivo, para evitar de esta manera el sesgo de género.

${ }^{2}$ Este artículo se deriva de una investigación de pregrado realizada por Silvana Aravena y Juan Pablo Contreras, con la guía de Mónica Palacios, para optar al título de terapeutas ocupacionales en la Universidad Andrés Bello de Chile.

${ }^{3}$ Terapeuta ocupacional. Corporación de Apoyo Psicosocial para Adultos Dependientes. Santiago de Chile, Chile. sarroyoa6@hotmail.com. (iD) ORCID: https://orcid.org/0000-0002-6242-3960

4 Terapeuta ocupacional. Centro de la Mujer Renca-Sernameg. ONG Redes de Orientación en Salud Social. Santiago de Chile, Chile. juanpacontreras8@gmail.com. (iD) ORCID: https://orcid. org/0000-0001-8276-8715

${ }_{5}$ Terapeuta ocupacional. Magíster en Psicología Social Comunitaria. Doctora en Salud, Bienestar y Calidad de Vida. Docente, Universidad de Santiago de Chile. Santiago de Chile, Chile. monica.palacios@usach.cl. (iD) ORCID: https://orcid.org/0000-0001- 5686-8237
} 


\begin{abstract}
This research proposes to recognize the secondary discourses of transsexual-transgender (trans) people, about their occupations, drawing attention to the diversity of their social practices. The methodology used was qualitative, descriptive-interpretative. In depth semi-structured interviews were conducted with four people over 18 years-old, who were selected intentionally. Discourse analysis was done by establishing three axes: self-recognition; discourse and occupation and social practice and its places of expression. The results show that transsexual subjects develop their identity from gender stereotypes referring to the man-woman dichotomy, where the masculine presents privileges over the feminine in society, thus observing that patriarchal heteronormativity is implicit in their occupations and in their daily life. Dominant and secondary discourses emerge, ranging from the good-acceptable and the bad-out of the norm.
\end{abstract}

\title{
KEY WORDS
}

transgender persons, gender norms, occupations, discourse

\section{RESUMO}

Esta pesquisa propõe reconhecer discursos subalternos de transexuais transgêneros (trans), sobre suas ocupações, tornando visível a diversidade de suas práticas sociais. A metodologia foi qualitativa, descritiva-interpretativa. Entrevistas semiestruturadas, em profundidade, foram realizadas com quatro pessoas maiores de 18 anos, selecionadas intencionalmente. A análise do discurso foi realizada, estabelecendo três eixos: autoreconhecimento; discurso e ocupação; prática social e seus lugares de enunciação. Os resultados mostram que sujeitos transexuais desenvolvem sua identidade a partir de estereótipos de gênero referentes à dicotomia homem-mulher, em que socialmente o masculino apresenta privilégios sobre o feminino. Assim, ele percebe que a heteronormatividade patriarcal está implícita em suas ocupações e em sua vida cotidiana. Deste modo, surgem discursos dominantes e subalternos, que vão desde o bem-aceitável e o mau fora da norma.

\section{PALAVRAS-CHAVE}

pessoas transgênero, normas de gênero, ocupações, discurso

\section{Introducción}
"Que un acto sea desviado o no depende entonces de la forma en que los otros reaccionan ante él (...) la desviación no es una cualidad intrínseca al comportamiento en sí, sino la interacción entre la persona que actúa y aquellos que responden a su accionar" (Becker, 2009, p.34)

Lo desviado se entenderá como la desviación de las normas sociales, por tanto, aquello que aparece desviado en un periodo histórico determinado, no lo es en otro momento y lugar (Becker, 2009). Los movimientos de diversidad sexual muestran aquello que se pudiera pensar como desviado, cuestionando, interpelando y ayudando a superar la dualidad de género y sexo; así, señalan y relevan a la diversidad como parte del ejercicio de la ciudadanía (Movi- 
miento de Integración y Liberación Homosexual -Movilh, 2015).

Desde una Terapia Ocupacional social y crítica, se considera que cada persona debe ser reconocida y respetada por su diversidad (Pino \& Ulloa, 2016). Esto supone una posición consciente, política y disciplinar frente a la posibilidad de reproducir relaciones desde la supremacía de masculinidades culturalmente dominantes, o producir relaciones transformadoras y contra-heteronormativas. De esta manera, se intenta reconocer discursos de aqueIlxs Ilamadxs desviadxs, como sujetxs sociopolíticxs, comprendiendo y valorando la desviación como diversidad, tal como lo concibe Becker (2009). En este sentido, se plantea la temática trans, referida a personas identificadas como transexuales ${ }^{6}$ o transgénero ${ }^{7}$ , con miras a visibilizar y romper barreras sociales para aquellxs descalificadxs socialmente como coliparadx ${ }^{8}$, coquet $x^{9}$ y desviadx, y pertenecientes a la comunidad lesbiana, gay, transexual, bisexual e intersexual (LGTBI+). Se busca además visibilizar que, en lo cotidiano, la construcción de identidad y la participación se dan desde la diver- sidad, a pesar de que la sociedad tiende a rechazar la diferencia.

Históricamente, la exclusión de la diferencia ha determinado formas de relaciones de poder y control social (Foucault, 1995). En Chile, esto se representa en políticas públicas o marcos legales sesgados, que desfavorecen la inclusión desde la mirada de los derechos humanos; lo anterior es respaldado por los estereotipos y estigmas que las prácticas sociales otorgan a las diferencias. En el país se está en deuda de avanzar en enfoques diferenciales para las políticas públicas, la Agenda 2030 para el desarroIlo sostenible afirma que la igualdad de género no solo es un derecho humano, también es imprescindible para alcanzar los objetivos de desarrollo; sin embargo, no basta con reconocer que los roles, derechos, obligaciones, recursos e intereses de mujeres y hombres se determinan cultural y socialmente de manera desigual, si se sigue entendiendo el género de manera binaria. De esta manera, la actual legislación invisibiliza, excluye y violenta a las personas trans, inhibiendo con ello una participación ciudadana plena, que les permita liberarse de aquello que se considera normal (Movilh, 2015).

\footnotetext{
${ }^{6}$ Mujer transexual: persona nacida con un sexo biológico masculino e identificada legalmente como hombre, pero que siente ser mujer desde temprana edad. El proceso y la transición médica, psicológica y social vividos por estas mujeres para adecuar el cuerpo a su identidad de género es denominado HaM (Hombre a Mujer). (Nathional Geographic , 2017).

- Hombre transexual: persona nacida con un sexo biológico femenino e identificada legalmente como mujer, pero que siente ser hombre desde temprana edad. El proceso y la transición médica, psicológica y social vivido por estos hombres para adecuar el cuerpo a su identidad de género es denominado MaH (Mujer a Hombre). (Nathional Geographic, 2017).

7 Transgénero: se utiliza para denominar a personas cuya identidad de género no concuerda con su sexo biológico, que fue designado al nacer de acuerdo con la genitalidad. También se refiere a conductas y grupos que se diferencian de las identidades de género binarias (hombre o mujer). (Nathional Geographic, 2017).

${ }^{8}$ Forma despectiva de referirse a hombres con características femeninas, travestis y transexuales, haciendo referencia a no tener vergüenza.

${ }^{9}$ Adjetivo que puede utilizarse en femenino o en masculino, para referirse a alguien esmerado en su apariencia personal y en su forma de expresión verbal y no verbal, con tal de parecer más atractivo e interesante.
} 
La sociedad normalizadora busca mecanismos de control que determinan como actuar, como relacionarnos, como caminar, etc.; de esta manera, se reprimen prácticas sociales alternativas y el ejercicio liberador de la ciudadanía. Se puede apreciar que las ocupaciones comunmente aceptadas pueden ser parte de lo que Foucault (1995) denomina control social y que, por tanto, estén enmarcadas en un sistema económico capitalista y patriarcal, que de manera hegemónica asigna roles de género de acuerdo al sexo biológico. Lo anterior refuerza directa o indirectamente los estereotipos masculinos y femeninos; invisibiliza, excluye y violenta a quienes no se acogen a tales estereotipos; niega la diversidad sexual y de género, y, finalmente, dificulta a muchas personas la posibilidad de reconocerse a sí mismas como distintas. Así, para ellas, la interacción con el contexto se traduce en falta de oportunidades, discriminación, vulneración de derechos y limitación de la participación en ocupaciones que no concuerdan con lo socialmente aceptado.

A pesar de que durante la represión en dictadura emergieron discursos de resistencia, subversivos y a su vez subalternos, como los de Pedro Lemebel ${ }^{10}$ y las Yeguas del Apocalipsis ${ }^{11}$, Ixs sujetxs diversxs surgen ahora como un grupo poco reconocido y subalternizado, que busca enfrentar las limitaciones sociales que pueden derivarse, precisa- mente, de identidades poco aceptadas o invisibilizadas (Bidaseca, 2010) por la heteronormada sociedad chilena.

La arraigada práctica conservadora y la herencia patriarcal postdictadura han permeado el difícil camino al ejercicio pleno de derechos por parte del eje LGTBI+. Como lo expresa Garrido (2016), el Chile de los años 90 se debatía contra la derogación del artículo 365 del Código Penal, el cual sancionaba la sodomía y defendía el ideal de la familia heteronormada. Recién en el año 2015, a través de la lucha de los movimientos LGTBI+, se logró la promulgación de la Ley Antidiscriminación 20.609, o "Ley Zamudio", y de la Ley 20.830 de Acuerdo de Unión Civil, que permite que personas de un mismo sexo puedan realizar un contrato de unión que reconoce y protege al conviviente. Sin embargo, siguen existiendo situaciones que denotan un alto grado de violencia y discriminación de género y sexo, con resistencia activa de grupos religiosos y políticos de gran poder en el país (Montecino, 1984).

Durante el año 2015, las denuncias y casos por homofobia y transfobia en Chile aumentaron un $8,8 \%$ respecto al 2014, la tendencia se ha mantenido al alza. Los 258 casos y denuncias del 2015 corresponden al $15,8 \%$ del total de episodios discriminatorios registrados desde el 2002 (Movilh, 2015). En

\footnotetext{
${ }^{10}$ Pedro Segundo Mardones Lemebel fue escritor, cronista y artista plástico chileno, activista de los movimientos de liberación homosexual. Su obra escrita aborda los temas de la marginalidad chilena, utilizando para ello algunas referencias autobiográficas. Nació el 21 de noviembre de 1952 y falleció el 23 de enero de 2015 (Yeguas del Apocalipsis, 2018).

11 El colectivo artístico Yeguas del Apocalipsis fue conformado por Pedro Mardones Lemebel (19522015) y Francisco Casas Silva (1959) en Santiago de Chile, entre los años 1987 y 1997. Siendo activos en la oposición a la dictadura, participaron en la lucha por los derechos humanos y han sido parte sustantiva del surgimiento de movimientos sociales contemporáneos (Yeguas del Apocalipsis, 2018).
} 
el 2018, aumentaron en un $44 \%$ las denuncias y abusos basados en la orientación sexual o la identidad de género, acumulando durante ese año un total de 698 casos, la cifra anual más alta conocida hasta ahora y que acumula el $22 \%$ del total de los 3.137 atropeIlos contabilizados en 17 años; así lo arrojó el XVII Informe anual de los derechos humanos de la diversidad sexual y de género. Los 698 casos se dividen en: 3 asesinatos; 58 agresiones físicas o verbales perpetradas por civiles desconocidos por las víctimas; 16 casos de abusos policiales; 28 hechos de discriminación laboral; 37 episodios de exclusión educacional; 102 movilizaciones o campañas homo/transfóbicas; 271 situaciones de marginación institucional; 17 denegaciones de derechos en espacios públicos o privados; 72 actos de violencia comunitaria (familiares, vecinos, amigos, conocidos); 92 declaraciones de odio, y 2 abusos en el terreno de la cultura, los medios o el espectáculo (Movilh, 2019).

Estos antecedentes de violencia y discriminación se difunden por parte de los medios de comunicación de forma sesgada, desinformada, muchas veces violentando derechos. De acuerdo con lo señalado por el Movimiento de Integración y Liberación Homosexual -Movilh, en el año 2015 la policía y los medios de comunicación se refirieron a las personas afectadas por vulneraciones y crímenes de odio por el nombre y sexo asignado al nacimiento y no por su nombre social, pasando por alto su identidad de género; resulta así que la ignorancia, los prejuicios y el trato discriminatorio contra las personas transexuales persisten, incluso, luego de que fallecen (Movilh, 2015).
Frente a estas discriminaciones, es necesaria una Terapia Ocupacional política y social, con perspectiva crítica, de género, y que valore la diversidad (Guajardo \& Galheigo, 2015; Simó, 2016); siendo relevante que la profesión se introduzca en la temática trans como ámbito de actuación y, de esta manera, romper con los estereotipos de género y su expresión en ocupaciones para hombres y otras para mujeres. Ello posibilita levantar la idea de un discurso trans de las ocupaciones como formas diversas de inclusión, es decir, ocupaciones que transiten liberadas de los roles de género establecidos por la herencia patriarcal de la colonización territorial y cultural, que constituyen características dominantes de lo masculino por sobre lo femenino (Bidaseca, 2010). Frente a los discursos dominantes de género y sexo, se busca rescatar los discursos subalternizados de la comunidad trans:

Los discursos no deben ser considerados como conjunto de signos, sino como prácticas que obedecen a reglas determinadas (...) en toda sociedad la producción del discurso está a la vez controlada, seleccionada y redistribuida por un cierto número de procedimientos, que tienen por función conjurar los poderes y peligros (Gabilondo, 1990, p 101).

El discurso de las ocupaciones puede ser entendido de diferentes maneras: como práctica social, concepto que la define como el componente fundamental del mundo social; como formas de decir y hacer en un tiempo y lugar definidos, o constituidas por sentidos, significados y materialidades que anteceden a las personas y a las estructuras 
sociales. Así, tanto la acción personal como la capacidad de las instituciones de moldear el mundo social serían el resultado del despliegue de las prácticas (Angenot, 2012; Miramón, 2013). De esta manera el discurso, entendido como práctica social pone en evidencia la existencia de discursos dominantes (Iñiguez, 2003) y heteronormativos, y, de manera contra hegemónica, de discursos subalternos (Zehar, 2006), entendiendo lo subalterno como relativo a los grupos excluidos de las sociedades, discriminados debido a su raza, etnia, clase social, capacidad funcional, género, orientación sexual o religión.

Los discursos con relación al género se manifiestan también en aspectos dinámicos del carácter de las ocupaciones en la vida cotidiana de las personas. En el decir de Pino y Ulloa (2016), "ocupación como expresión histórica diversa, como praxis en constante transformación, como potencial humano que va mutando a través de las tensiones y luchas sociales, a través de los cambios de paradigmas y el desarrollo de las fuerzas productivas" (p. 426).

Desde estas ideas sobre ocupación, se quieren poner en tensión, por un lado, los efectos potenciales de las ocupaciones en la opresión y, por otro, en el aporte a la lucha por el reconocimiento de la diversidad sexual y de género, como una práctica diversa que derrote estereotipos referentes al quehacer de una orientación sexual o de género. De esta manera, se busca atender al hecho de que las personas transexuales y transgénero nos desafían con su diversidad, frente a los discursos tendientes a homogeneizar y estandarizar las realidades.
Al respecto, Honneth (1997) ayuda a comprender y valorar las diferencias desde la Teoría del Reconocimiento, pues plantea que las formas señaladas de vulneración o negación de derechos y exclusión social producen una grave restricción de la autonomía, generando, en quien lo sufre, un sentimiento de no ser sujetx moralmente válidx e igual a otrxs. Desde la perspectiva señalada, se reconoce la diversidad de géneros como un principio esencial en la construcción de una humanidad diversa y democrática. Por otro lado, visibilizar la dominación entre géneros y sexualidades que produce la opresión heteronormada es el primer paso para la emancipación. Una humanidad diversa y democrática requiere de personas dispuestas a ser diferentes de quienes han sido, para ser reconocidas en la diversidad y vivir en la democracia genérica (Lagarde, 1996).

El propósito de la investigación fue analizar los discursos -entendidos como prácticas sociales- de personas trans, en cuanto a sus ocupaciones. Los objetivos específicos fueron: distinguir las diversas maneras en que Ixs participantes se reconocen como personas trans; identificar y describir las posiciones de los discursos de las personas trans participantes, con respecto a sus ocupaciones; identificar y describir los discursos subalternos de las personas trans participantes, con respecto a sus ocupaciones. 


\section{Métodos}

Se asumió una metodología cualitativa, de carácter descriptivo - interpretativo, dado que se abordan aspectos subjetivos de las vivencias de personas que son parte de un grupo social invisibilizado y estigmatizado. Se buscó, a través de esta metodología, aportar a esa voz desde la desestigmatización, en el entendido de que tal posición ubica en el reconocimiento de las condiciones de sumisión y dominación de dicho grupo.
La selección de participantes se realizó con una técnica muestral intencionada, de manera que las personas cumplieran con los requisitos necesarios para el estudio; entre ellos: reconocerse como personas trans femeninos/masculinos, mayores de 18 años, con y sin participación en organizaciones o movimientos sociales. En la tabla 1 se resumen las características de las personas participantes de la investigación.

Tabla 1. Características de las personas participantes.

\begin{tabular}{|ll|}
\hline Sujetx & $\begin{array}{l}\text { Descripción } \\
\text { trans femenina, } 45 \text { años, comuna de Conchalí, educación media completa, } \\
\text { trabaja en empresa de envasado como operaria, ex participante de } \\
\text { organizaciones LGTBI+. }\end{array}$ \\
\hline E2 & $\begin{array}{l}\text { Trans masculino; } 30 \text { años; comuna de la Florida; geógrafo con estudios de } \\
\text { género, identidad y derechos humanos; activista LGTBI+; trabaja como } \\
\text { geógrafo en el Cajón del Maipo. }\end{array}$ \\
\hline E3 & $\begin{array}{l}\text { Trans femenina, } 42 \text { años, Santiago Centro, trabajadora sexual, defensora de } \\
\text { los derechos humanos, ex participante de organizaciones LGTBI+. }\end{array}$ \\
\hline E4 & $\begin{array}{l}\text { Trans masculino, 22 años, comuna de Nuñoa, educación superior incompleta, } \\
\text { trabaja en empresa familiar. }\end{array}$ \\
\hline
\end{tabular}

Fuente: elaboración propia.

La información fue producida a través de entrevistas en profundidad, semi-estructuradas, con cada uno de Ixs participantes. Las entrevistas se desarrollaron en torno a los siguientes temas: relatos de vida, reconocimiento-identidad como persona trans, discursos de las personas trans con respecto a sus ocupaciones, prácticas sociales en la vida cotidiana.

Los resultados emergen desde un análisis del discurso, donde las temá- ticas centrales se desglosan en tres dimensiones, según Fairclough (2003): en primer lugar, en el texto, haciendo referencia al discurso literal del sujetx, del cual emergen las categorías al agrupar las similitudes entre los discursos de Ixs participantes. De allí emergieron categorías y subcategorías relacionadas con los ejes de análisis previos: auto-reconocimiento; discurso trans; ocupaciones y prácticas sociales.

En segundo lugar se encuentra la 
práctica discursiva, que se refiere al análisis intertextual, alineando las categorías en comparación con la conceptualización utilizada en el marco teórico, los antecedentes y el contexto desde el cual se está hablando. A partir de allí, se identificaron posiciones de habla desde las cuales emanaron los diferentes discursos.

Finalmente, como tercera dimensión está la práctica social, que alude y evalúa la posición del sujetx o de habla, relacionada directamente con las características propias de Ixs participantes. En esta dimensión se realizó una discusión de los hallazgos con algunos de los conceptos presentes en los discursos de Ixs participantes.

\section{Consideraciones éticas}

Se realizó un tratamiento confidencial de la información, respetuoso de las historias y relatos confiados a Ixs investigadores. Se informó a Ixs participantes, a través de un documento escrito y también de manera verbal, sobre el tipo de investigación, sus propósitos y alcances. La participación fue voluntaria. Lxs participantes firmaron un consentimiento informado.

\section{Resultados}

\section{Categorías y subcategorías del estudio}

Las categorías principales son:

Autoreconocimiento. A partir de lo que significa para Ixs sujetxs pertenecer al mundo trans, en sus historias de vida, aparece este concepto que alude al re- conocerse o autoidentificarse o no como tal. Este concepto ayuda a reflexionar acerca de los procesos sociales e intersubjetivos vinculados con la construcción de las identidades personales (De la Masa, 2010).

Discurso trans. Se relaciona con lo que comparten Ixs sujetxs en la entrevista, sus diálogos y relatos sobre sus experiencias e historias de vida. Así mismo, estos discursos serán entendidos, según Iñiguez (2003), como prácticas sociales, ya que se construyen con base en los significados que los mismxs sujetxs les dan al interactuar con y en sus contextos culturales. Este concepto, al igual que el anterior, se distingue por la relación que establecen Ixs participantes con sus propios contextos y cómo esta, a su vez, crea significados diversos para Ixs sujetxs.

Ocupación y práctica social. Se refiere a las actividades de la vida cotidiana. Estas pueden ser o no significativas y pueden ser consideradas o no ocupaciones por Ixs sujetxs. A su vez, se relaciona con las prácticas sociales aceptadas socialmente para las personas trans, las que practican debido a una posición de sujetx subalternizadx y que fueron referidas por Ixs participantes.

En cuanto a las posiciones de sujetx o de habla, aparecen tres:

Sujetx fortalecidx. Se refiere a aqueIlxs sujetxs que participan dentro de la sociedad, de manera organizada y activa. De igual forma, se reconoce y se visibiliza como trans y realiza actividades consideradas desde Ixs sujetxs como una ocupación.

Sujetx subalternx. Alude a quien participa en sociedad, pero depende de las 
condiciones del contexto si se reconoce o se visibiliza como trans; a pesar de esto, lucha por una posición de poder.

Sujetx invisible. Se refiere al sujetx que participa dentro de la sociedad sin reconocerse como trans ni demostrar su identidad u orientación sexual, para no ser catalogado negativamente. Sujetx que apoya la lucha pero no participa en ella.

A continuación, se integran las categorías analizadas desde las posiciones de habla.

\section{Posición de sujetx fortalecidx, autoreconocimiento y discurso dominante}

A partir de esta posición de sujetx, se hace referencia a la categoría autoreconocimiento. Tello (2011) refiere que los seres humanos se constituyen en relación con otrxs sujetxs y, producto de esa interacción, configuran su subjetividad en tanto identidad de género y comienzo de la transición:

...en abril me di cuenta que yo era transexual, en abril del año pasado, y ahí me dediqué un poco a averiguar un poco de en verdad qué era, qué se hacía, y una vez que ya estuve como así cien por ciento seguro de lo que iba a hacer, le conté a mi hermana, después a mis papás, mis abuelos, tíos, primos; entonces fue todo como, eso lo conté como en octubre, en noviembre me empecé a inyectar $y \ldots$ (E4)

Para contextualizar la cita, E4 es el más joven de los entrevistados; según refiere, pertenece a un estatus socioeconómico alto y su hermana mayor tiene orientación homosexual-lesbia- na. De acuerdo con lo anterior, se puede explicar su posicionamiento y reconocimiento como trans desde lo histórico familiar, como primer y fundamental agente de socialización; en este caso, le otorga seguridad y comprensión frente a su diversidad, dado que en su familia ya habían vivenciado una situación similar con su hermana. Es decir, como su contexto más cercano y cotidiano acepta y respeta las diversidades, promueve una identificación segura frente a un contexto macro amenazante. En conjunto, se podría justificar también desde las palabras de Honneth (1997), donde Ixs sujetxs se identifican a partir del reconocimiento que tiene el contexto social sobre ellxs.

Por otra parte, al tener los recursos necesarios para acceder a la intervención hormonal, se promovió un transitar facilitado, a diferencia de Ixs demás entrevistadxs, de bajos niveles socioeconómicos y con dificultades para acceder a un tratamiento en el sistema de salud chileno. La inequidad mencionada dificulta el proceso de identificación como sujetx diferente y promueve situaciones de discriminación. Cabe destacar que E4 nació en un período histórico contemporáneo, donde las diversidades se han comenzado a visibilizar y hay una lucha por sus derechos ciudadanos, lo que también promueve su posición fortalecida.

Con respecto a la categoría discurso dominante, se muestra la siguiente cita respecto al hecho de salir a la calle:

no pa ná, de hecho ahora me siento mucho más seguro de cuando salía cuando era Valentina. (E4) 
Este relato alude al pensamiento normativo de las masculinidades, ya que expresa el sentimiento de seguridad frente a una identidad masculina por sobre la femenina; como reflejan las palabras de Montecino (1984), desde una concepción machista que otorga privilegios sociales a la identidad masculina, cuando socialmente se desconoce que hubo transición. Lo anterior es un fiel reflejo del intrínseco pensamiento heteronormado de la cultura chilena, como si el hombre no tuviera derecho a temer, plantado con seguridad desde un posicionamiento de poder frente a cualquier expresión femenina.

\section{Posición de sujetx subalterna y discurso trans}

Bajo esta posición, se analizará la categoría discurso trans, específicamente la subcategoría discurso dominante, donde la pregunta referida es, si se considera parte de la comunidad LGBTI.

... cuando a mí me preguntaron: ¿tú siempre te sentiste hombre? Yo no tengo otra forma de decir que sí, siempre me sentí hombre, pero si yo tengo que pensar que ser hombre es lo que dice la sociedad que significa ser hombre, yo no quiero ser eso, no pertenezco al típico hombre del patriarcado, aquel que tiene un montón de privilegios, porque de hecho no los tengo, porque los privilegios vienen desde lo fálico, y evidentemente yo no tengo un falo... (E2)

De acuerdo con la posición de habla y la categoría seleccionada, se da cuenta de cómo los discursos de las personas trans se constituyen a partir de la norma establecida como dominante, generando sujetxs con privilegios (en la posición de masculinidad), en desmedro de aquellxs despojadxs de los mismos (en la posición de feminidad). Como bien señala Iñiguez (2003), los discursos se construyen a partir de la praxis de Ixs sujetxs a través de la interacción y la socialización con el contexto, las cuales están determinadas en el tiempo y el espacio que van definiendo, a su vez, una época concreta.

...no fue tan difícil en mi familia, sí podría decir que fue difícil en el colegio, onda bulliyng todo el rato (...), o sea, yo tuve compañeros que me decían: -te vamos a violar para hacerte mujer-, por ejemplo. (E2)

Así mismo, el contexto en el cual habitan las personas trans está caracterizado desde sus inicios por una cultura machista y heteronormada, y si tomamos nuevamente las palabras de Montecino (1984), es una sociedad donde el hombre se posiciona por sobre la mujer. Es por esto que E2, a pesar de sentirse hombre, no se quiere sentir como el hombre que establece la norma, en una posición de poder por sobre lo femenino.

En relación a la categoría ocupación y prácticas sociales, la pregunta se refiere a las ocupaciones socialmente aceptadas o rechazadas:

... el trabajo sexual es una situación más por falta de oportunidades laborales, específicamente, y tiene que ver con que, por más que vos estudiés y seás una profesional, siempre será el tema ser una trans. Entonces, por ejemplo, si yo fuera odontóloga, cuantas 
personas se irían a arreglar una dentadura con una mujer trans... hay un estigma, o sea, aun siendo profesional, tengo que estar parada en una avenida... (E3)

...hay un cartel que había en una marcha y salía una chica travesti que decía: -claro empresario, de noche me buscas para llevar a tu casa o a un motel, pero en el día no me quieres dar trabajo- ...la sociedad le pide a las chiquillas trans que hagan ese tipo de trabajo, no les deja otras opciones y les empieza a angostar más el camino para llegar a él. (E2)

De acuerdo con la posición de habla de E2 y E3, se puede visualizar que es el mismo contexto social el que está limitando la elección y la realización de una ocupación a Ixs sujetxs que se visibilizan como trans dentro de la sociedad; como menciona Rogero (2000), ser diverso se refiere a no aceptar el modelo heteronormado, impuesto por la mayoría. Además, bajo este mismo término y reflejado en las citas anteriores, se encuentran situaciones de violencia, discriminación, desigualdad, segregación y limitación a ejercer como ciudadano pleno dentro de una sociedad.

Parece interesante la afirmación de E2, cuando menciona cómo el contexto influye, delimita y permite ciertos accionares y actividades laborales en las personas, es decir, que el contexto social condiciona el actuar y la vivencia de cada sujetx. Heller (1972) lo menciona cuando habla desde el actuar permitido en la vida cotidiana, además de referirse a las jerarquías sociales y a los posicionamientos hegemónicos.
Cabe destacar las connotaciones negativas ligadas a la marginalidad que se atribuyen al trabajo y el comercio sexual; su ejercicio tiene una atribución de género, ya que se asume como una labor hecha por mujeres, desconociendo parte de la diversidad masculina que, de igual forma, podría ejercerlo. Sin embargo, dentro del pensamiento social se atribuye a lo femenino una posición inferior que cubre necesidades masculinas.

\section{Posición de sujetx invisible, y ocupación y prácticas sociales}

Esta posición da cuenta de quienes participan dentro de la sociedad sin reconocerse como trans y viven su proceso ocultando sus vivencias. Limitándose a ocupaciones aceptadas socialmente, están sometidxs a las reglas sociales y solo se muestran cuando están entre iguales:

...en trabajos que sean muy marcados de hombre, y yo creo que tengo cero posibilidad de ser carpintero o constructor o cualquier cosa así. (E1)

Em... yo creo que más trabajo social, quizás, y trabajos que conllevan una connotación más femenina por la delicadeza... mmm... aún así, pudiendo ser que conlleve algo más fuerte; pero trabajos así como ultra masculinos sobre todo así de peso, de fuerza: no, cero posibilidad. (E1)

Lo anterior está planteado desde el tránsito a ser hombre y cumplir con los estereotipos masculinos de fuerza, respecto a las ocupaciones posibles. Implica una falta de oportunidades desde 
contextos y sujetxs heteronormadxs, partícipes de él. Bajo una perspectiva de derechos, esto se traduce en una situación de discriminación; es decir, el que Ixs sujetxs se reconozcan, se visibilicen dentro de esta realidad nacional, promueve que se expongan a situaciones donde se vulneran sus derechos, por tanto, a esta posición subyace el temor a ser rechazadxs y agredidxs.

\section{Discusión}

Los hallazgos señalados en el apartado anterior se pueden discutir con relación a la pluralidad de posiciones de habla que emergen y cómo estas muestran contradicciones entre voces fortalecidas, subalternas e invisibles, incluso en un mismx sujetx, a lo largo de la narración de sus vivencias. A partir de las categorías emerge el transitar, con sus dificultades y complejidades para las personas y sus contextos; el transitar entre géneros y sexualidades sin duda tensiona las normas sociales naturalizadas y dominantes acerca del género y la sexualidad (Lamas, 2000).

La dicotomía hombre-mujer, como relación binaria, no da cabida a la diversidad, va atrapando y trata de asimilar eso diferente. Para afrontar el binarismo es necesario el reconocimiento (Honneth, 1997) de esx otrx, de lo diverso como posibilidad de existencia; también es necesario posibilitar el autoreconocimiento, ya que como sujetxs transitando también han sido atrapadxs en la heteronormatividad y el binarismo. Esta condición se ve determinada por las situaciones cotidianas, culturales, históricas, políticas y económicas, que afectan las posibilidades y oportunidades para un transitar pleno.
El imperativo social es que hay que decidir: ieres trans-hombre o trans-mujer?, ¿transitas hacia hombre o hacia mujer? Esta demanda social por definirse en el binarismo tensiona y horada las identidades, obligando, muchas veces, a tomar decisiones bajo presión, con todo lo que implica asumir un rol de género hombre o mujer; esta situación ha sido vivida por las personas entrevistadas. La sociedad comprendida binariamente es una herencia cartesiana que pesa en el día a día e inhibe posibilidades otras de existencia, de ser/hacer.

Desde ahí, el reconocimiento implica, entre otras cosas, volver sobre el conocimiento que se da por sentado acerca de lo que significa ser humano, y del lugar que ocupan las sexualidades y los géneros. Más allá del respeto por la diversidad, es una comprensión profunda y compleja de la condición humana. Lo anterior supone una conciencia de los mecanismos de reproducción social, una alerta de cómo en la vida cotidiana (Heller, 1972; Bourdieu \& Passeron,1979) se van naturalizando acciones, pensamientos y omisiones que empujan a decidir: blanco o negro, hombre o mujer; que obligan a categorizarse como seres humanos en representaciones de sí dentro de un orden binario que, además, dé certezas. Por otro lado, romper con los mecanismos de reproducción supone la incertidumbre, aquello desconocido que surge como amenaza, la creencia de que al desarmar los binarismos, viene el caos.

Entendiendo que las ocupaciones normadas y normalizadoras de hombre y mujer oprimen la pluralidad y reproducen del control social, entonces: ¿Cómo podríamos Ixs terapeutas ocupa- 
cionales ocuparnos de ello? Un camino es replantearnos la actividad humana como pluralidad, en oposición a la actividad humana desde la normatividad -actividades para hombres y otras para mujeres-; de esta manera, romper con el binarismo de las actividades definidas por género. Esto nos obliga a pensar, promover e intervenir desde una Terapia Ocupacional inclusiva en los diferentes contextos, para favorecer escenarios que visibilicen las pluralidades, las socialicen y se sensibilicen con ocupaciones diversas y emancipadoras.

En este sentido, se destacan dos publicaciones recientes en la profesión, con perspectivas de género y feministas. La primera de ellas profundiza en los procesos subjetivos de mujeres jóvenes que han sido madres adolescentes, sus interseccionalidades y las implicaciones en la investigación e intervención en Terapia Ocupacional (Cortina, 2019). La segunda aborda de manera crítica la función económica de las ocupaciones feminizadas no remuneradas y propone la idea de una economía feminista desde las ocupaciones del cuidado (Grandón, 2019). Ambos artículos se alinean con la propuesta aquí planteada, en el sentido de cuestionar el sentido de las ocupaciones y la actuación de la Terapia Ocupacional, pues aún los estereotipos de género producidos por el machismo están presentes en la sociedad contemporánea y, a pesar de estar más abierta a aceptar y tolerar la diversidad, se sigue creyendo que el hombre debe ser proveedor de una familia, mientras la mujer es desvalorizada, no tiene voz, está destinada a ocupar lugares subalternos, a gestar y hacer labores domésticas.
Los escenarios contemporáneos posibles para promover las pluralidades están dados por condiciones de vida que sumergen a las personas en el individualismo, la pérdida de sentidos (Lipovetsky, 1986; Bauman, 2003), el consumo exacerbado y el hedonismo omnipresente en las relaciones humanas. Estas condiciones inundan de estereotipos de belleza, deseados y deseables, que llevan a quienes se sienten diferentes, desviados, a experimentar que no encajan, a vivir las intersecciones y pluralidades de su vida como una carga, no como una riqueza.

Reafirmando lo planteado, se van generando ideales de cómo se es trans y cómo se debe ser trans. Allí se ponen en tensión realidades de personas trans que, al mismo tiempo, viven en pobreza y no tienen los recursos para acceder, por ejemplo, a una cirugía de reasignación de sexo; aunque este tipo de procedimientos resultan fundamentales para algunas personas, también invisibilizan culturas y formas de ser y estar diversas. Entonces, las intersecciones de sexualidad, género, condición económica, cultura, etc., quedan atrapadas en estereotipos de belleza y de género dominantes, en un contexto sociohistórico donde los ideales de imagen y de éxito resultan inalcanzables para muchxs.

Para la Terapia Ocupacional es central comprender las ocupaciones y a Ixs sujetxs como diversos. Implica tomar conciencia de que a las ocupaciones o actividades de la vida cotidiana se les sigue otorgando un valor según roles de género femeninos o masculinos $y$, en consecuencia, cada persona asume de manera irreflexiva el realizar 
actividades de acuerdo con su identidad de género, según lo socialmente aceptado.

\section{Conclusiones}

Los discursos de las personas trans participantes respecto a sus ocupaciones, entendidos como prácticas sociales, denotan un predominio por la clasificación y la categorización binaria; no hay espacio para el transitar. El no pertenecer al binarismo genera incertidumbre y empuja de manera imperativa a adoptar en la sociedad las diferencias de género y su expresión en lo que hemos denominado: ocupaciones binarias.

Se distinguen diversas maneras de reconocerse como persona trans. Algunxs de quienes transitan de hombre a mujer o de mujer a hombre se reconocen desde estereotipos femeninos o masculinos, relevando las identidades dominantes respecto a ser hombre o mujer. Sin embargo, otrxs no se identifican en estas posiciones binarias y en el proceso de tránsito manifiestan no querer reproducir roles patriarcales.

Se identifican tres posiciones de sujetx o habla en los discursos de las personas trans participantes respecto a sus ocupaciones: fortalecido, subalterno e invisible. En los discursos subalternos respecto a sus ocupaciones, especialmente en las referidas al trabajo, se manifiestan tensiones y contradicciones entre ocupaciones binarias y ocupaciones que transgreden el binarismo en los roles de género.

Las prácticas sociales de Ixs participantes tratan de integrase a aquellas socialmente aceptadas, sin embargo, muchas veces se sienten empujados a prácticas rechazadas o ilícitas, como el comercio sexual, por no responder a los estereotipos de género tradicionales. Al ser al ser parte de un universo desviado, marginadxs y condicionadxs a escoger esta ocupación, se les asocia a situaciones que reproducen prejuicios frente a las personas trans, entre ellos, que es la única ocupación que pueden realizar. Ser parte de la diversidad desviada de la norma social machista, heteronormada y binaria, trae como consecuencia situaciones de discriminación, exclusión, marginación, vulneración de derechos y limitaciones para realizar ocupaciones que sean del interés de Ixs sujetxs.

La investigación tuvo como limitación el escaso tiempo para el desarrollo del trabajo de campo, especialmente para el desarrollo de las entrevistas en profundidad y la generación de la confianza necesaria con Ixs participantes. Esto se reflejó en la disminución del número de entrevistadxs, respecto al que se había proyectado inicialmente. Por otro lado, en el momento en que se realizó el estudio (2015-2016) había escasez de antecedentes desde Terapia Ocupacional.

Respecto a los alcances, se abren posibilidades para problematizar aún más las ocupaciones binarias. Así mismo, se podrían realizar otras investigaciones sobre ocupaciones que se desvíen de la heteronormatividad, o que profundicen en las relaciones entre ocupaciones y género, en el lugar del género en la construcción de las ocupaciones o en el papel de las ocupaciones en las identidades de género. Estos estudios pueden ayudar a transitar críticamente hacia una Terapia Ocupacional reconocedora 
de las diferencias, que promueva ocupaciones humanizantes, plurales, diversas, sin preconcepciones de género moralizantes, aportando con ello a la construcción de un discurso como palabra y acción que dignifique la diferencia y la desviación de aquello Ilamado norma.

\section{Referencias}

Angenot, M. (2012). El discurso social. Ios límites históricos de lo pensable y lo decible. Buenos Aires: Siglo XXI.

Bauman, Z. (2003). Modernidad líquida. México: Fondo de Cultura Económica.

Becker, H. (2009). Outsiders: hacia una sociología de la desviación. México: Siglo XXI.

Bidaseca, K. (2010). Perturbando el texto colonial. Los Estudios (Pos)coloniales en América Latina. Buenos Aires: Editorial SB.

Bourdieu, P. \& Passeron, J. (1979). La reproducción: elementos para una teoría del sistema de enseñanza. Barcelona: Editorial Laia.

Cortina, L.A. (2019). Subjetividades, interseccionalidades e implicaciones en la investigación e intervención ocupacional con madres adolescentes. Revista Ocupación Humana, 18(2), 41-53. doi: https://doi.org/10.25214/25907816.227

De la Masa, L. (2010). Actualizaciones del concepto hegeliano de reconocimiento. Veritas, (23), 67-94. doi: https://dx.doi. org/10.4067/S0718-92732010000200004

Fairclough, N. (2003). El análisis crítico del discurso como método para la investi- gación en Ciencias. En R. Wodak \& M. Mayer (Eds.), Métodos de análisis crítico del discurso (pp. 179-203). BarceIona: Gedisa.

Foucault, M. (1995). ¿Qué es la crítica? [Crítica y Aufklärung]. Daimon Revista Internacional de Filosofía, (11), 5-26.

Gabilondo, A. (1990). El discurso en acción: Foucault y una ontología del presente. Madrid: Anthropos, Editorial del Hombre.

Garrido, J. (2016) Sexualidades bajo control. Discursos y representaciones sobre la diversidad sexual en el Chile de los 90. Historias que vienen. Revista de Estudiantes de Historia UDP, 7, 34-49. Recuperado de http://socialesehistoria. udp.cl/wp-content/uploads/2016/05/ Revista-Historias-que-vienen-7.-Texto-completo.pdf

Grandón, D. (2019). Función económica de las ocupaciones feminizadas no remuneradas: una crítica desde la economía feminista. Revista Ocupación Humana, 18(2), 54-67. doi: https://doi. org/10.25214/25907816.228

Guajardo, A. \& Galheigo, S. (2015). Reflexiones críticas acerca de los derechos humanos: Contribuciones desde la terapia ocupacional Latinoamericana. World Federation of Occupational Therapists Bulletin, 71 (2), 73-80. doi: https://doi.org/10.1179/14473828 15Z.00000000023

Heller, A. (1972). Historia y vida cotidiana: aportación a la sociología socialista. México: Grijalbo.

Honneth, A (1997). La lucha por el reconocimiento. Por una gramática moral 
de los conflictos sociales. Barcelona: Crítica.

Iñiguez, L. (2003). Análisis del discurso, Manual para las Ciencias Sociales. Barcelona: UOC.

Lagarde, M. (1996). Género y feminismo. Desarrollo humano y democracia. España: Horas y Horas.

Lamas, M. (2000). Diferencias de sexo, género y diferencia sexual. Cuicuilco, 7(18), 1-25.

Lipovetsky, G. (1986). La era del vacío. Ensayos sobre el individualismo contemporáneo. Barcelona: Anagrama.

Miramón, M. (2013). Michel Foucault y Paul Ricoeur: dos enfoques del discurso. $\mathrm{La}$ Colmena,78, 55-57.

Montecino, S. (1984). Mujeres de la Tierra. Santiago de Chile: CEM.

Movimiento de Integración y Liberación Homosexual -Movilh. (2015). Violencia contra personas lesbianas, gays, bisexuales, transexuales e intersexuales (LGBTI) en América. Santiago de Chile: Movilh. Recuperado de https:// www.movilh.cl/biblioteca/estudios/

Movilh. (2019). XVII Informe anual de los derechos humanos de la diversidad sexual y de género. Santiago de Chile: Movilh. Recuperado de https://www. movilh.cl/un-44-aumentan-los-casos-ydenuncias-por-homofobia-y-transfobiaen-el-ano-mas-fructifero-para-los-derechos-trans/

Nathional Geographic. (enero de 2017). Redefinir el género. National Geografhic en Español, 40(1), p.V.
Pino, J. \& Ulloa, F. (2016). Perspectiva crítica desde Latinoamérica: hacia una desobediencia epistémica en Terapia Ocupacional contemporánea. Cadernos Brasileiros de Terapia Ocupacional, 24(2), 426. doi: https://doi.org/10.4322/01044931.ctoARF0726

Rogero, J. (2000). Trabajar con la diversidad para romper las desigualdades. En: Congreso Construir la escuela desde la diversidad y para la igualdad, conferencia llevada a cabo en el Congreso, CEAPA, CCOO, FETE-UGT, STE, CGT, MRP y MCEP, Madrid, España. Recuperado de http://www.nodo50.org/igualdadydiversidad/rogero.htm

Simó, S. (2016). Terapia ocupacional, cultura y diversidad. Cadernos Brasileiros de Terapia Ocupacional, 24(1), 163-171. doi: http://dx.doi.org/10.4322/01044931.ctoRE0677

Tello, F. (2011). Las esferas de reconocimiento en la teoría de Axel Honneth. Revista de Sociología, (26), 45-47.

Yeguas del Apocalipsis. (2018). Las Yeguas del Apocalipsis: Pedro Lembel y Francisco Casas. Chile: Yeguas del Apocalipsis. Recuperado de http://www.yeguasdelapocalipsis.cl

Zehar, N. (2006). Spivak o la voz de subalterno. Rebelión. Recuperado de http://www. rebelion.org/noticias/2006/11/41618.pdf 